\title{
A Constraint on Antigravity of Antimatter from Precision Spectroscopy of Simple Atoms*
}

\author{
S. G. Karshenboim*** \\ Max-Planck-Institut für Quantenoptik, Garching, 85748 Germany \\ Mendeleev Institute for Metrology, St. Petersburg, 190005 Russia \\ Received November 28, 2008
}

\begin{abstract}
Consideration of antigravity for antiparticles is an attractive target for various experimental projects. There are a number of theoretical arguments against it but it is not quite clear what kind of experimental data and theoretical suggestions are involved. In this paper we present straightforward arguments against a possibility of antigravity based on a few simple theoretical suggestions and some experimental data. The data are: astrophysical data on rotation of the Solar System in respect to the center of our galaxy and precision spectroscopy data on hydrogen and positronium. The theoretical suggestions for the case of absence of the gravitational field are: equality of electron and positron mass and equality of proton and positron charge. We also assume that QED is correct at the level of accuracy where it is clearly confirmed experimentally.
\end{abstract}

PACS numbers: $04.80 . \mathrm{Cc} ; 11.30 . \mathrm{Er} ; 12.20 . \mathrm{Fv}$

DOI: $10.1134 / \mathrm{S} 1063773709100028$

Key words: Equivalence principle, CPT symmetry, Galactic gravitational field, quantum electrodynamics, precision spectroscopy.

\section{INTRODUCTION}

After producing cold antihydrogen atoms via the recombination of positrons and antiprotons [1,2] a number of possibilities to experimentally test CPT invariance including precision spectroscopy and gravitation have been intensively discussed. In particular, a question of experimental check for a possibility for antigravity of antiparticles rose for new consideration.

It is absolutely clear that the very suggestion of antigravity for antimatter contradicts general relativity (GR). While physics of matter objects in free fall cannot be distinguished from the zero gravity case (as long as any gradient effects can be neglected), an antigravitating particle will clearly recognize the gravitation field. ation:

Here we refer to antigravity for the following situ-

(i) the inertial masses of particles and antiparticles are the same;

(ii) the gravitation masses, understood, e.g., as the related coefficients for Newtonian gravity at rest for long distances (where the Newtonian gravity strongly

\footnotetext{
* The article was translated by the author.

${ }^{* *}$ E-mail: savely ·karshenboim@mpq.mpg.de
}

dominates over any general relativity effects), have the same absolute values for particles and antiparticles;

(iii) the particle-particle and antipartic-le-antiparticle gravitational interaction is attractive, while the particle-antiparticle one is repulsive.

In particular, that means that in any given [weak] gravitation field a particle and its antiparticle experience forces, equal in their absolute values and opposite in directions. A concise review on the issue of antigravity can be found, e.g., in [3].

General relativity sets an even stronger direct constraint on a possibility of antigravity. Observation of various effects beyond Newtonian gravity [4] proves that gravitation is due to a tensor field which (in contrast to a vector field for electromagnetic forces) produces only attraction and no repulsion. Any speculation on the so-called fifth force considers only small corrections beyond the tensor forces [5] and can be ignored in the case of a $100 \%$ effect such as antigravity.

Still, because of the sensitivity of this issue, the experimental community would prefer to have some more straightforward constraint, for which it would be clear which experimental data and theoretical suggestions are involved in ruling out antigravity.

Here we present such a consideration. 


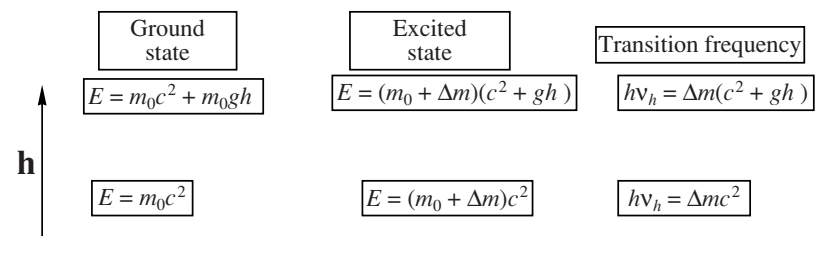

Fig. 1. Derivation of the gravitational red shift.

Prior to a detailed examination, we have to make two remarks. First, we remind that there are two kinds of gravitation-related effects. Some are sensitive to a certain long-range difference of the gravitation potential $U(\mathbf{r})$, while the other are sensitive to the local field strength $\mathbf{g}=-\nabla U(\mathbf{r})$.

Second, when one "tests" gravity, a few kinds of interactions are commonly considered. Some, like true gravitation, couple to the mass $m$, while the others couple to some charges. The charge is additive and it is the same, e.g., for the ground and excited states of an atom. We do not consider here interactions coupled to such quantities as magnetic moments, which are neither additive, nor conserved. The mass and charges are regular. Mass is a statedependent property in atomic physics since different states possess different binding energy. This energy is directly related to emission/absorption photon energy. On the contrary, any conserved charge is state-independent and it is the same for any state in the same atom. The magnetic moment (or a similar quantity) is state-dependent, but it depends on the internal structure of a related state.

In particular, antigravity means a coupling to mass. Otherwise, we have to suggest that all the gravity is a force coupled to a baryonic and/or leptonic charge. The latter indeed changes its sign for antimatter, but the results for tests of the equivalence principle and GR in general indeed rule out this option $[4,5]$.

\section{THE RED SHIFT OF THE PHOTON FREQUENCY AS THE BLUE SHIFT OF THE CLOCKS}

The idea of our derivation is based on a study of the red shift in atoms and we remind here how one can derive the red shift expression with 'minimal' and clearly formulated suggestions. (Note, once we consider a possibility for antigravity of antiparticles we cannot blindly rely on GR.)

Let us consider ground and excited states of an atom in two positions at different gravitational potentials. Different states have different masses such as

$$
\Delta m c^{2}=h \nu_{0},
$$

where $\nu_{0}$ is the photon frequency. The recoil effects for emission or absorption are neglected and the related correction does not change the result of our consideration.

With masses being different for the ground and excited states, the gravitation energy is also different (see Fig. 1)

$$
\Delta E=\Delta m \Delta U
$$

by a value which is related to a correction for the emitted/absorbed photon frequency

$$
h \Delta \nu=\Delta m \Delta U \text {. }
$$

Within this consideration the shift mentioned is for the frequency of a clock, while a photon traveling through the time-independent gravitation field does not change its frequency. ${ }^{1}$ The derivation above, which follows [6], shows that the gravitational red shift is not a specific property of GR, but rather a generic property of any relativistic theory of gravity since it is supposed to reproduce Newtonian gravity and special relativity as crucial limits.

What is called the gravitational red shift of the photon frequency within GR depends on a choice of 4 -coordinates. Depending on how the time is defined (or how clocks are synchronized in space), it may be a shift in photon frequency or in clock frequency (or in both). The observable effect is a certain mismatch in photon frequency while communicating between two clocks at different gravitational potentials. In particular, in our consideration, instead of the red shift of the photon going "upward" we observe a blue shift of the transition frequency in the upper clock in respect to the bottom clock (see, e.g., [6]). The mismatch of the frequency sent to an upper potential is equivalent to the red shift by a conventional value:

$$
\frac{\Delta \nu}{\nu_{0}}=\frac{\Delta U}{c^{2}} \text {. }
$$

Usually the red-shift experiments are organized as relative and differential. We can either compare the red shift of two different clocks, or we can compare the experimental red shift to a theoretical calculation, based on a measurement of the free fall acceleration with bulk matter or atomic beams. In the former case we check universality of the red shift for different traveling frequencies and different frequency sources. Actually, this is the universality of the gravitational effect on all local clocks that allows to redefine the

\footnotetext{
${ }^{1}$ Actually, it is rather natural to suggest (once we assume antigravity of antimatter) that the matter and antimatter are affected by gravity in opposite directions, while "pure neutral objects" such as positronium atoms and photons are not affected by gravity at all. That immediately leads to a contradiction with gravitational deflection of light, which is well established experimentally.
} 
time in any way we like. The latter case confirms that the gravitation acting on matter governs photon behavior as well. In both cases the gravitational red shift is a comparison of a certain frequency of a clock measured at two different positions.

Looking for a non-universality of the red shift in different clocks we have a certain advantage. Usually, one also has to deal with a number of motional effects, which partly or completely cancel gravitational effects under certain conditions. Studying a differential red shift (i.e., a difference in red shifts of two clocks), the motional effects, which are always universal, do not contribute.

\section{POTENTIAL OF A GRAVITATIONAL ATTRACTOR FOR AN ORBITAL FRAME}

Let us check another option. Instead of measuring at two location we can measure a ratio of two frequencies at one location at a certain distance $r$ from a point-like attractor and determine the same at an infinite distance from gravitating masses theoretically. In this case, we can take advantage of considering a weak field, which may accumulate a large value of $\Delta U$ over a large distance, while the field by itself is negligible for any $\mathrm{g}$-related effects.

A circular rotation of a probe body with velocity $v$ around mass $M$ deals with the field (acceleration)

$$
g=\frac{v^{2}}{r}=G \frac{M}{r^{2}}
$$

and the difference of potential (in respect to an infinitely remote point) is equal to

$$
\Delta U(r)=-G \frac{M}{r}=-v^{2} .
$$

That in particular means that if we need to estimate any $U(r)$-related effect, we can do a proper estimation once we know the velocity of rotation. The noncircularity of the orbit cannot change the order of magnitude for the estimation.

Let us consider a scenario with a slowly rotating body at a circular orbit. Effects due to the local gravitational force are small as long as the distance is large. However, the potential in respect to an infinitely remote zero-gravity point is not small. If we position our probe clocks on this body, we will observe a gravitational shift with respect to infinitely remote points as

$$
\frac{\Delta \nu}{\nu_{0}}=-\frac{v^{2}}{c^{2}} .
$$

In our laboratory experiments we deal with a few motions which produce relatively large values of $v^{2} / c^{2}$. For our motion around the Sun we have $v^{2} / c^{2} \simeq 10^{-8}$, while the Solar System moving around the center of our galaxy supplies us with a value $v^{2} / c^{2} \simeq 10^{-6}$ [7]. We do not expect that a noncircularity of the orbits can change the estimations. We note that the related field is very small, but the potential difference is large enough. Indeed, the universe is not so simple as consisting of a gravitating center which determines solar and Earth motion mentioned. However, a good approximation is that most of the universe is homogeneously distributed around us and its gravitational effect vanishes. (In any case, any other motion induced by a gravitational potential at a larger scale can be treated in the same way.)

If we consider only matter with the equivalence principle for gravitational and inertial masses $\left(m_{\mathrm{g}}=\right.$ $m_{\mathrm{i}}$ ), the shift is universal and cannot be locally detected since all clocks are shifted in the same way.

\section{COMPARISON OF HYDROGEN AND ANTIHYDROGEN AND POSITRONIUM CLOCKS}

Once we suggest antigravity for antimatter and include antimatter clocks into consideration, the matter and antimatter clocks would be shifted in opposite directions. Once we know the ratio of two frequencies at $r=\infty$, we can compare it with a local value and prove or disprove antigravity.

There is no antimatter clock available for the moment, but a kind of a "neutral" clock is available. Once we suggest antigravity for antimatter, the positronium is a system with no gravity at all.

In particular, assuming the antigravity once should expect

$$
\nu(r)=\nu_{0} \times \begin{cases}1+\frac{U(r)-U(\infty)}{c^{2}} & \text { for } \mathrm{H} \\ 1 \quad \text { for Ps, } & \\ 1-\frac{U(r)-U(\infty)}{c^{2}} & \text { for } \overline{\mathrm{H}}\end{cases}
$$

Meanwhile, the frequencies $\nu_{0}$, unperturbed by gravity, are the same for the same transition in the hydrogen and antihydrogen atoms, while the ratio for positronium and hydrogen is calculable (see below).

We note that the positronium $1 s-2 s$ transition $[8,9]$ was measured with a high accuracy as well as the $1 s-2 s[10]$ and some other [11] transitions in hydrogen. And that can be applied to experimental searches for the antigravity option.

The ratio of the $1 s-2 s$ frequencies in hydrogen and positronium can be calculated at zero gravity. In the leading approximation (Schrödinger equation for a particle in the Coulomb field) this ratio is equal to the ratio of the related reduced masses which are 


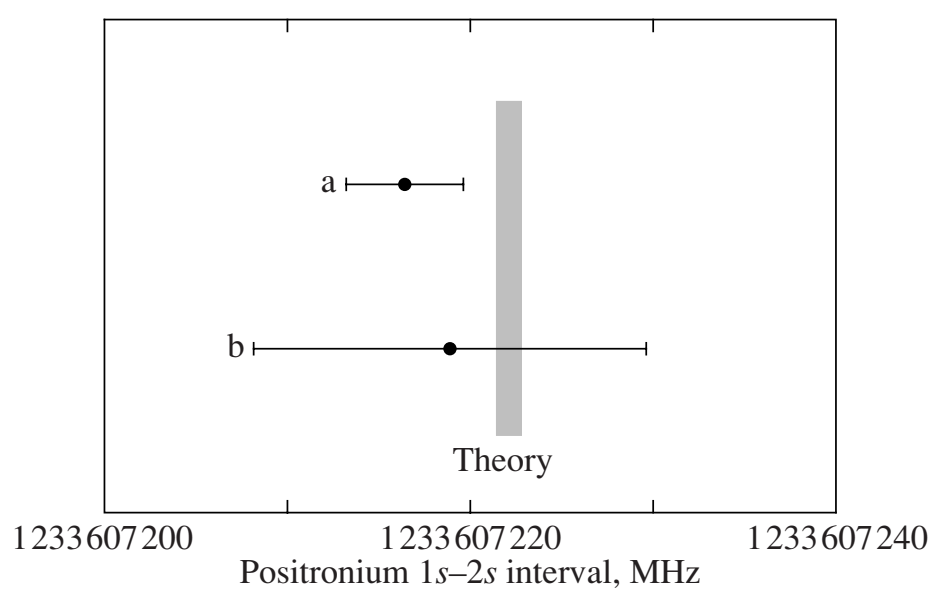

Fig. 2. Determination of the $1^{3} S_{1}-2^{3} S_{1}$ interval in positronium. The references here are: a is from [8] and b is from [9]. The plot is taken from [12].

$$
\begin{gathered}
m_{\mathrm{R}}(\mathrm{Ps})=\frac{m_{e}}{2}, \\
m_{\mathrm{R}}(\mathrm{H})=\frac{m_{e}}{1+m_{e} / m_{p}},
\end{gathered}
$$

where $m_{e}$ is the electron mass and $m_{p}$ is the proton mass. A number of corrections are known (see, e.g., [12]) and the theoretical result for the unperturbed frequency ratio has the form

$$
\begin{gathered}
\frac{\nu_{0}^{\mathrm{Ps}}\left(1^{3} S_{1}-2^{3} S_{1}\right)}{\nu_{0}^{\mathrm{H}}\left(1^{3} S_{1}-2^{3} S_{1}\right)} \\
=\frac{1}{2}\left(1+\frac{m_{e}}{m_{p}}\right) \times F\left(\alpha, m_{e} / m_{p}\right),
\end{gathered}
$$

where $F\left(\alpha, m_{e} / m_{p}\right)$ stands for known QED corrections (see $[12,23])^{2}$

The theoretical ratio agrees with the experimental ratio within an uncertainty of a few parts in $10^{-9}$.

Technically, instead of considering the frequency ratio for hydrogen and positronium transitions, we compare positronium experimental results for $\nu^{\text {th }}\left(1^{3} S_{1}-2^{3} S_{1}\right)$ with theory based on the value of the Rydberg constant obtained from the hydrogen spectroscopy (see, e.g., [13]). A comparison of theory and experiment is plotted in Fig. 2. The theoretical result (see, e.g., [12])

$$
\nu_{0}^{\text {th }}\left(1^{3} S_{1}-2^{3} S_{1}\right)=1233607222.2 \pm 0.6 \mathrm{~Hz}
$$

perfectly agrees with experiment $[8,9]$

$$
\begin{gathered}
\nu^{\mathrm{a}}\left(1^{3} S_{1}-2^{3} S_{1}\right)=1233607216.4 \pm 3.2 \mathrm{~Hz}, \\
\nu^{\mathrm{b}}\left(1^{3} S_{1}-2^{3} S_{1}\right)=1233607219 \pm 11 \mathrm{~Hz} .
\end{gathered}
$$

\footnotetext{
${ }^{2}$ There are also corrections due to the proton structure which are of marginal interest for our consideration.
}

In the absence of gravity, theory should agree with experiment (once the calculations are correct). However, in the presence of gravity and under the suggestion of antigravity of antimatter we should introduce the gravitational corrections. That will affect the hydrogen theory and shift the value of the Rydberg constant from hydrogen spectroscopy by $\Delta U / c^{2}$.

Such a correction should obviously shift a theoretical value and produce a discrepancy between theory and experiment at the level of one ppm. That immediately rules out any room for antigravity once we accept that the galactic field which determines rotation of the Solar system does not include any exotic component. That is at the level of a few hundred standard deviations.

Here we considered a rotation around the center of galaxy as only motion. If we consider some more intergalaxy-scale motions [7], that will add an additional gravitational source and will increase the effect. However, that will not change the order of magnitude of the effect.

If we address only the solar gravity and use the orbiting velocity of Earth, we arrive at a limitation at the level of $4 \sigma$ which opposes a possibility for antigravity, but is not too convincing.

\section{QED AND ANTIGRAVITY}

The theoretical ratio is obtained once we assume that the Coulomb interaction of an electron and a positron is the same as that of a proton and an electron. The other assumption applied is that the inertial masses of an electron and a positron are the same.

We can rely on the fact that there are many direct or indirect tests for these two facts at the level of interest $\left(10^{-6}\right)$. However, we prefer just to mention that obviously any 'exact' compensation between the 
gravitational red shift and inequalities of masses and charges could happen only if they are of gravitational origin, which can be checked in many other ways.

In any case an agreement for $g-2$ experiments with uncertainty at the level of a few parts in $10^{11}$ confirms that an old-fashioned Dirac equation, which suggests the identity of mass and charge values for electron and positron, is still a reasonably good approximation. The results for $g-2$ are of two kinds: there are (i) measurements on slow electrons and positrons which confirm that both agree with each other [14] and with theory and (ii) an experiment on ultrarelativistic beams of electrons and positrons [15], which produces the same result for either beams.

To be more specific with comparison of the most accurate electron $g-2$ experiment [16] with theory [17], we note that for a successful application of theory one needs a value of the fine structure constant $\alpha$. Instead of comparison of $g-2$ theory and experiment one can equivalently compare different alpha's. The most accurate $\alpha$ is from electron $g-2[16,17]$, the second in accuracy method is for atomic interferometry with cold rubidium [18] and caesium [19] beams. These results agree (see [13] for detail) at the level of uncertainty much lower than $10^{-6}$.

These arguments mean that we can really rely on equality of electron and positron masses and on universality of elementary charge as the value of charge of electron, positron, proton etc.

As it was mentioned, from the theoretical viewpoint antigravity is rather unlikely. Can the method considered here be used for any other constraints? It seems that not in any straightforward way. E.g., if the $1 s-2 s$ transition in antihydrogen were measured with about the same accuracy as in hydrogen, which means an uncertainty at the level of a part in $10^{14}$, combining that with a $10^{-6}$ red shift we could claim universality of free fall for matter and antimatter at the uncertainty level of $10^{-8}$. However, that would not be very useful. That would be correct only for "massrelated' gravity. Only the latter interferes with the red shift since the excited and ground states have different mass. Once we suggest an exotic coupling due to, e.g., baryonic charge, that would affect 'mechanical' free fall (i.e., a real fall of a bulk body or an atom), but not its small contribution to the red shift, since the baryonic charge in ground and excited states is the same.

Nevertheless, in general, considering a particular model of modification of gravity, some constraint on its parameters could be set and the transitions of interest are transitions measured in hydrogen, positronium, muonium and antiprotonic helium (see below) and transitions in antihydrogen which are to be measured in some future.

In principle, there may be some reservations about the application of the galaxy gravitation field that is produced by matter and a substantial amount of the dark matter. Still, consideration of the gravitation of the Sun at the Earth orbit is also sufficient to rule out antigravity. In any case, the uncertainty due to the nature of the Sun gravity cannot be larger than for the Earth gravity where the antihydrogen antigravity experiments are supposed to be realized.

As already mentioned, we could also consider muonium and antiprotonic helium. Muonium consists of a heavy nucleus, which is a positive muon (an antiparticle), and a light orbiting particle, which is an electron. Antiprotonic helium consists of a conventional nucleus (the $\alpha$ particle) and an orbiting antiproton and an electron. The latter can be treated as a perturbation. The related results are quite accurate $[20,22]$, but it is hard to interpret them in a model-independent way for an antigravity search. In a conventional case we deal only with inertial mass of a compound system, which is a sum of component masses corrected due to their binding energy. The latter is related to the whole two-body system and well defined. Shares of a binding energy related to each component are not observable. If we assume antigravity for antiparticles, we should split the binding energy between the material and antimaterial components of the system. Such a share can be indeed assigned ad hoc, but since it is not observable in any other effect, there is no way to prove any particular assignment. However, we note that the pair of atoms mentioned, namely muonium and antiprotonic helium, have a complementary structure: both consist of two components (once we neglect an electron in antiprotonic helium) with a particle and an antiparticle present; one component is light, the other is heavy and both options are realized: heavy particle and heavy antiparticle.

In principle, the electromagnetically bound twobody system is just the simplest example of an electromagnetic problem for a particle or antiparticle bound by the electromagnetic field produced by other particles or antiparticles.

In particular, we can consider resonance transitions between quantized levels of particles and antiparticles in the magnetic field, which we indirectly mentioned when we referred to $g-2$ experiments as a kind of clock. Indeed, the field is produced by matter and it is a difficult question how to split a "material" and "antimaterial" part of the binding energy of an antiparticle in the magnetic field. Considering the transitions in terms of a search for the gravitational red shift, which is potentially much higher than the 
Effects, uncertainties, sensitivities



Fig. 3. Fractional values of gravitational effects versus uncertainty and sensitivity of various precision measurements (see also [25]).

uncertainty in some experiments, we can find a controversy considering electrodynamics with electrons and positrons in the free falling frame. Further consideration needs indeed a model.

However, if we like to consider electrodynamics, we should introduce the electromagnetic field and thus the energy levels mentioned above, e.g., the Landau levels are to be determined by the field value. Because of the gravitational red shift, the result of the field action on a particle in the case of antigravity could potentially depend on the origin of the field (whether it is created by matter or antimatter) and kind of a probe particle (whether it is a particle or antiparticle) at the ppm level in laboratory experiments. Antigravity would make classical electrodynamics quite problematic.

In the case of an antiparticle at a classically produced electromagnetic field we arrive at the same problem-how to split the binding energy between the material field source and a particle of antimatter, which is crucial for antigravity. It seems that the most consistent way would be to distribute it according to the masses of the objects. E.g., for equal charges the share of binding energy between two inertial masses $m_{i}$ could be proportional to $m_{i} /\left(m_{1}+m_{2}\right)$. In such a case muonium would behave in the gravitational field nearly as an atom of antimatter (since $m_{\mu} \gg$ $m_{e}$ ), while in antiprotonic helium-4, the gravitational mass would be about $60 \%$ of the inertial mass $(0.6=$ $(4-1) /(4+1))$. For the antiprotonic helium-3, also studied experimentally [20] and theoretically [21], the gravitational mass would be $50 \%$ of the inertial mass.

We remind that the experimental uncertainty of the $1 s-2 s$ in muonium [22] is $4 \mathrm{ppb}$, while the combined uncertainty of antiprotonic helium spectroscopy [20] is about $60 \mathrm{ppm}$ and it is going to be substantially improved. Theory has sufficient accuracy (see, e.g., 12, 21, 23). Applying both we should also rule out the antigravity option considering rotation around the center of galaxy.

\section{CONCLUSIONS}

Concluding the paper, we summarize that we have presented straightforward arguments against a possibility of antigravity based on a few simple theoretical assumptions and experimental data. The data are: astrophysical data on rotation of the Solar System in respect to the center of our galaxy and precision spectroscopy data on hydrogen and positronium. The theoretical assumptions for the case of absence of gravitational field are: equality of electron and positron mass and equality of proton and positron charge. We also assume that QED is correct at the level of accuracy where it is clearly confirmed experimentally.

For future activity, we have to mention one more option for a possible model-independent limitation on antigravity. If a measurement of the $1 s-2 s$ transition in positronium can be improved by an order of magnitude (or a measurement of this transition in hydrogen will be performed at the same accuracy), one should be able to observe such a clear signature of antigravity as annual variation of the ratio of the $1 s-2 s$ transitions in hydrogen and positronium (or antihydrogen) due to change in distance between the Sun and the Earth which changes by about 5 million kilometers during the year. That is related to a change in the gravitational potential in fractional units by

$$
\frac{\Delta U\left(r_{\max }\right)-\Delta U\left(r_{\min }\right)}{c^{2}} \simeq 3.2 \times 10^{-10} .
$$

The shift of all material clocks (including hydrogen's) is a blue shift for $r=r_{\max }$ in respect to $r=r_{\min }$, while a positronium clock would experience no shift and the antihydrogen clocks would be red shifted.

There is a number of experiments with low uncertainty or with a high sensitivity and a summary is presented in Fig. 3. 
Due to importance of positronium spectrum and annihilation of free and bound positrons study of positronium annihilation line from the Galactic Center region can also deliver some constraints on antigravity. At present the accuracy (as observed by SPI/INTEGRAL [24]) is not sufficient but may be improved in future missions.

\section{ACKNOWLEDGMENTS}

The work was in part supported by DFG (under grant № GZ 436 RUS 113/769/0-3) and RFBR (under grants № 08-02-91969, 06-02-16156, 08-02-13516).

Stimulating discussions with M. Fujiwara, J. Walz, D.A. Varshalovich, R.A. Sunyaev, S.I. Eidelman, and V.G. Ivanov are gratefully acknowledged.

\section{REFERENCES}

1. M. Amoretti, C. Amsler, G. Bonomi, et al., Nature 419, $456(2002)$.

2. T. Aoyama, M. Hayakawa, T. Kinoshita, and M. Nio, Phys. Rev. Lett. 99, 110406 (2007); Nucl. Phys. B796, 184 (2008).

3. R. de Beauvoir, F. Nez, B. Cagnac, et al., Phys. Rev. Lett. 78, 440 (1997).

4. E. Churazov, R. Sunyaev, et al., Mon. Not. R. Astron. Soc. 357, 1377 (2005).

5. P. Cladé, E. de Mirandes, M. Cadoret, et al., Phys. Rev. Lett. 96, 033001 (2006).

6. M. I. Eides, H. Grotchb and V. A. Shelyuto, Phys. Rep. 342, 63 (2001).

7. K. Danzmann, M. Fee, and S. Chu, Phys. Rev. A 39, 6072 (1989).

8. H. Dehmelt, R. Mittleman, R. S. Van Dyck, Jr., and P. Schwinberg, Phys. Rev. Lett. 83, 4694 (1999).

9. M. S. Fee, A. P. Mills, Jr., S. Chu, et al., Phys. Rev. Lett. 70, 1397 (1993).

10. M. Fischer, N. Kolachevsky, M. Zimmermann, et al., Phys. Rev. Lett. 92, 230802 (2004).
11. G. Gabrielse, N. S. Bowden, P. Oxley, et al., Phys. Rev. Lett. 89, 213401 (2002); Phys. Rev. Lett. 89, 233401 (2002).

12. D. Hanneke, S. Fogwell, and G. Gabrielse, Phys. Rev. Lett. 100, 120801 (2008).

13. M. Hori, A. Dax, J. Eades, et al., Phys. Rev. Lett. 87, 093401 (2001).

14. S. G. Karshenboim, Phys. Rep. 422, 1 (2005).

15. S. G. Karshenboim, arXiv:0811.1009v1 [gr-qc] (2008).

16. V. I. Korobov, Phys. Rev. A 77, 042506 (2008).

17. F. Maas, B. Braun, H. Geerds, et al., Phys. Lett. A 187, 247 (1994).

18. R. K. Mittleman, I. I. Ioannou, H. G. Dehmelt, and N. Russell, Phys. Rev. Lett. 83, 2116 (1999).

19. P. J. Mohr, B. N. Taylor, and D. B. Newell, Rev. Mod. Phys. 80, 633 (2008).

20. M. Niering, R. Holzwarth, J. Reichert, et al., Phys. Rev. Lett. 84, 5496 (2000).

21. M. M. Nieto and T. Goldman, Phys. Rep. 205, 221 (1991).

22. L. B. Okun, G. K. Selivanov, and V. Telegdi, Usp. Fiz. Nauk 169, 1141 (1999) [Phys. Usp. 42, 1045 (1999)].

23. L. B. Okun, K. G. Selivanov, and V. I. Telegdi, Am. J. Phys. 68, 184 (2000).

24. S. Schlamminger, K.-Y. Choi, T. A. Wagner, et al., Phys. Rev. Lett. 100, 041101 (2008).

25. P. B. Schwinberg, R. S. Van Dyck, Jr., and H. G. Dehmelt, Phys. Rev. Lett. 47, 1679 (1981).

26. C. Schwob, L. Jozefowski, B. de Beauvoir, et al., Phys. Rev. Lett. 82, 4960 (1999).

27. R. B. Tully, E. J. Shaya, I. D. Karachentsev, et al., Astrophys. J. 676, 184 (2008).

28. S. G. Turyshev, Euro. Phys. J. ST163, 227 (2008).

29. I. B. Vasserman, P. V. Vorobyov, E. S. Gluskin, et al., Phys. Lett. B 187, 172 (1987).

30. A. Wicht, J. M. Hensley, E. Sarajlic, and S. Chu, Phys. Scr. T 102, 82 (2002).

31. C. M. Will, Living Rev. Relativity 9, 3 (2006), arXiv:gr-qc/0510072. 